\title{
Outcomes of an integrated care pathway for concurrent major depressive and alcohol use disorders: a multisite prospective cohort study
}

Andriy V. Samokhvalov ${ }^{1,2,3,4^{*}}$ [D, Charlotte Probst ${ }^{3,8,9}$, Saima Awan ${ }^{1}$, Tony P. George ${ }^{1,2,4}$, Bernard Le Foll ${ }^{1,2,3,4,5}$, Peter Voore ${ }^{1,4}$ and Jürgen Rehm ${ }^{1,2,3,4,5,6,7,8,9}$

\begin{abstract}
Background: In 2013, an Integrated Care Pathway (ICP) for concurrent Major Depressive (MDD) and Alcohol Use (AUD) Disorders was developed at the Centre for Addiction and Mental Health (CAMH), Toronto, Ontario, Canada. The ICP was further implemented at 8 other clinical sites across Ontario (the DA VINCI Project) in 2015-2017. The goal of this study was to systematically describe and analyze the main clinical outcomes of the project.

Methods: Data on a non-randomized cohort of patients receiving ICP-based treatment were collected prospectively at nine clinical sites in a variety of clinical settings. Statistical methods: descriptive statistics, t-test, chi-square, ANOVA, generalized linear models.

Results: Two hundred forty-six patients were enrolled, 58.8\% males, mean age was 45.6 years, 170 patients received treatment at academic health centres (AHC), 49 - at community hospitals (CH) and 27 - in family health teams (FHT). There were no major differences in anamnestic parameters and depression severity between the three settings, but there were differences in baseline drinking patterns between subgroups ( $F=4.271, \mathrm{df}=2, p=0.015)$. Overall completion rate was $70.7 \%$ with no significant variation between settings $\left(x^{2}=3.35, d f=2, p=0.19\right)$. Treatment duration in AHC was the longest, and completion rates were the highest. There was a statistically significant and clinically meaningful reduction in the number of drinking days per week $(1.81, t=8.78, p<0.001)$. The cohort overall demonstrated significant and meaningful reduction in severity of cravings (Penn Alcohol Craving Scale: 4.42, $t=8.63, p<0.001$ ) and depressive symptoms (Quick Inventory of Depressive Symptomatology: 4.25, $t=11.26, p<0.001$ ). While some of the baseline patient characteristics and treatment parameters varied between the settings, the variation in clinical outcomes was mostly insignificant, though clinical improvement was more pronounced in academic setting and with individual therapy.

Conclusions: The study demonstrated that ICP is a feasible and effective treatment for concurrent AUD and MDD that delivers meaningful clinical improvement in a variety of settings. A randomized controlled study is needed to properly compare the treatment outcomes between ICP model and treatment as usual and to further explore the role of various factors on treatment outcomes.
\end{abstract}

Keywords: Concurrent disorders, Alcohol use disorder, Major depressive disorder, Care pathway, Pharmacotherapy, Psychotherapy, Integrated treatment

\footnotetext{
* Correspondence: andriy.samokhvalov@camh.ca

${ }^{1}$ Addictions Division, Centre for Addiction and Mental Health (CAMH), 33 Russell Street, Office T519, Toronto, ON M5S 2S1, Canada

${ }^{2}$ Institute for Medical Science, University of Toronto, Toronto, ON, Canada

Full list of author information is available at the end of the article
}

(c) The Author(s). 2018 Open Access This article is distributed under the terms of the Creative Commons Attribution 4.0 International License (http://creativecommons.org/licenses/by/4.0/), which permits unrestricted use, distribution, and reproduction in any medium, provided you give appropriate credit to the original author(s) and the source, provide a link to the Creative Commons license, and indicate if changes were made. The Creative Commons Public Domain Dedication waiver (http://creativecommons.org/publicdomain/zero/1.0/) applies to the data made available in this article, unless otherwise stated. 


\section{Background}

In 2013, an Integrated Care Pathway (ICP) for concurrent Major Depressive (MDD) and Alcohol Use (AUD) Disorders was developed at the Centre for Addiction and Mental Health (CAMH), Toronto, Ontario, Canada [1]. These two conditions were chosen as both of them are highly prevalent in Canada and worldwide [2,3] and often are comorbid with each other, which significantly complicates their effective treatment [4]. Both conditions are also associated with high socioeconomic burden [5, 6] and there is a lack of well-established evidence-based treatments for the treatment of concurrent MDD and AUD [7]. The ICP was created in order to address this systemic shortcoming and after a short pilot stage showed promising clinical results $[8,9]$. In 2015 the ICP received support through a joint funding program Adopting Research To Improve Care (ARTIC) in order to implement this ICP at multiple clinical sites across the province of Ontario. The 22-month project was named DA VINCI (Depression and Alcoholism: Validation of an Integrated Care Initiative) and was completed in January 2017 with the ICP fully implemented at nine clinical sites including $\mathrm{CAMH}$.

The goal of this study was to summarize the main clinical outcomes of the implementation of ICP in a variety of clinical settings and to explore the determinants of clinical improvement in patients receiving treatment within the ICP paradigm.

Objectives of the study were to evaluate the treatment completion rate as well as the changes in severity of depressive symptoms, cravings for alcohol and patterns of drinking over the course of treatment.

The primary hypothesis was that patients receiving treatment through the ICP would have a retention rate significantly higher than the retention rate in patients who received treatment as usual (TAU) as previously reported for the central site $(\mathrm{CAMH}, 42.0$ and $30.9 \% 12$ and 16-week retention rates, respectively) [9].

Secondary hypotheses 1-3: There would be a significant reduction in 1) severity of depressive symptoms, 2) severity of cravings, and 3) weekly alcohol consumption levels over the course of ICP treatment.

\section{Methods}

Design: The study is observational in nature and had one prospective cohort of patients receiving ICP treatment at multiple sites. There was no control group. Patients were not randomized into treatment (convenience sample). Data were collected through a review of the clinical charts of all patients enrolled into the ICP since its inception in December 2013 and until September 30th, 2016. The latter time limit was set in order to ensure that all patients had at least 16 weeks to complete the ICP by the time the data collection was completed (January 20th, 2017). No post-treatment follow-up data were collected.

Eligibility criteria: Patients were considered eligible for the ICP when they had concurrent AUD and MDD and were never diagnosed with bipolar disorder. Cases of alcohol-induced depressive disorder were excluded. Patients were diagnosed with alcohol-induced depressive disorder if there was a clear temporal association between the onset of depressive symptoms and alcohol intoxication or withdrawal. Alcohol-induced depressive disorder was ruled out if the onset of depressive symptoms preceded the onset of excessive alcohol use, the symptoms persisted for a substantial period of time after the cessation of alcohol use or there was a clear history of non-alcohol-related depressive episodes as per DSM-5 diagnostic criteria and guidelines for differential diagnosis [10]. There were no age restrictions and patients with a wide variety of sociodemographic and clinical characteristics were included to ensure delivery of treatment to a broader population of eligible patients. Patients who required medical alcohol withdrawal management received it first and then were reassessed and enrolled into the project.

Setting: The project was carried out at 8 sites in Ontario in addition to $\mathrm{CAMH}$, or 9 in total. These sites included 2 academic health centres (AHC): Royal Ottawa Mental Health Centre, Toronto Western Hospital / University Health Network; 3 community hospitals $(\mathrm{CH})$ : Trillium Health Partners, North Bay Regional Health Centre, and William Osler Health System and 3 large family health teams (FHT): Hamilton FHT, Village FHT and Inner City FHT.

Intervention: The ICP model includes several crucial components: Pharmacotherapy for both MDD and AUD - treatment was provided by trained physicians who followed the manualized treatment algorithm, which included several antidepressants of different pharmacological classes (sertraline, fluoxetine, venlafaxine and mirtazapine) [11-13] and several medications for the treatment of AUD (naltrexone, acamprosate and topiramate) [7, 12, 14] (See Additional file 1 for details). The algorithm was based on several clinical measures to monitor patients' therapeutic responses and justify the changes of medications and/or their dosages [15, 16]. These measures included quick inventory of depressive symptomatology (QIDS) [17], Penn alcohol craving scale (PACS) [18] and patterns of drinking operationalized via the following four indicators: number of standard drinks consumed on weekly basis $(\mathrm{SD} / \mathrm{w})$, number of drinking days per week $(\mathrm{DD} / \mathrm{w})$, number of heavy drinking days per week $(\mathrm{HDD} / \mathrm{w})$, and the number of standard drinks consumed per drinking day (SD/DD). Standard drink was defined as any alcoholic beverage containing $13.6 \mathrm{~g}$ of ethanol as per the Canada's Low-Risk 
Drinking Guidelines [19]. Another important component of the ICP was a 16-week manualized psychotherapy protocol which combined empirically supported treatments for both MDD and AUD such as cognitive behavior therapy (CBT) and motivational interviewing [20]. Initial sessions (1-2) focused on building therapeutic alliance, introduction of the treatment model and motivational enhancement, sessions 3-5 focused on behavioral components and gradually transitioned to cognitive techniques in sessions 6-10. Starting with session 11 protocol focused on relapse prevention, strengthening and rehearsal of the skills acquired during treatment, preparation for transition into community $[1,8]$. Psychotherapy was provided weekly by trained clinicians in group or individual format depending on the setting. Also, where possible the ICP was supported by pharmacists and nurses who monitored patients' medication compliance and provided psychoeducation when necessary. Finally, all clinicians involved in the ICP were meeting on weekly basis to increase the cohesion and integrative collaboration of the clinical team.

Outcome measures:

1. ICP completion rates: the treatment was considered completed if patients had received at least 12 core ICP psychotherapy sessions while being enrolled into the pathway or, in few cases, when they have completed the psychotherapy manual in accelerated pace and demonstrated significant clinical improvement (achieved remission from both MDD and AUD).

2. Severity of depressive symptoms: Measured by 16-item Quick Inventory of Depressive Symptomatology (QIDS-SR 16 [17]) at baseline and biweekly throughout the treatment.

3. Severity of cravings was measured by Penn Alcohol Cravings Scale (PACS [18]) biweekly.

4. Drinking patterns: Self-reported at each biweekly clinical visit and included number of drinking days per week $(\mathrm{DD} / \mathrm{w})$, number of standard drinks per week $(\mathrm{SD} / \mathrm{w})$, number of standard drinks per drinking day $(\mathrm{SD} / \mathrm{d})$ and number of heavy drinking days (HDD) per week (HDD/w).

5. Severity of depressive symptoms and cravings and drinking patterns were measured at baseline and at the end of treatment (or last clinical visit for patients who dropped out).

Data collection: Data were extracted from clinical charts by three independent raters. Clinical characteristics included medical history, drug use history, alcohol use and AUD history, history of depressive symptoms and MDD, history of treatments for conditions, current drinking patterns and comorbidities. The data from 10 charts were extracted by two raters simultaneously in order to ensure reliable data extraction and inter-rater agreement was evaluated for key variables. Furthermore, data extracted by one rater were randomly checked by other raters for potential extraction errors. In cases of disagreement, the principal investigator reviewed the issue and a consensus on extraction was reached.

Statistical methods: All analyses were performed in $\mathrm{R}$ v.3.3.1 [21]. Means and proportions were used to describe the baseline and end-of-treatment characteristics of the sample, which were then compared using paired t-test. ANOVA was used to compare the difference between subgroups of subjects based on the type of clinical settings (AHC, HC, FHT), and type of therapy they received (group or individual). Chi-square test for homogeneity was used for between-group comparisons of categorical variables. A series of linear models were calculated to evaluate the impact of setting and therapy format on clinical outcomes. All tests were bidirectional and based on alpha error of 5\%. For non-completers intent-to-treat approach was used [22]. Missing data were imputed using last observation carried forward [23].

Research ethics board: The study received approval from CAMH REB (protocol \#053-2016).

\section{Results}

\section{Patient characteristics}

A total of 396 patients with concurrent MDD and AUD were enrolled into ICP across the 9 sites including 44 patients who started their treatment before the DA VINCI project was funded (pilot group) and the 81 patients included into previous report [24]. Out of these, we have selected 246 patients who received treatment within the specified timeframe - 170 patients received treatment in academic health centres (AHC), 49 - in community hospitals $(\mathrm{CH})$ and 27 - in family health teams (FHT). Overall, $58.8 \%$ of the samples were men, with the $\mathrm{CH}$ subgroup having almost equal distribution of men and women, insignificantly higher proportion of men in AHC and lower - in FHT. Mean age was 45.6 years, there were no significant differences between subgroups $(\mathrm{F}=0.364, \mathrm{df}=2, p=0.695)$. More than half of patients were tobacco smokers (52.4\%); majority of them $(70.7 \%)$ used cannabis in the past month.

On average patients had 14.85 years of AUD history and 12.39 years of MDD history, with no significant difference between settings $(\mathrm{F}=0.47, \mathrm{df}=2, p=0.625$, and $\mathrm{F}=1.22, \mathrm{df}=2, p=0.299$, respectively). While severity of depressive symptoms as per QIDS did not vary much between subgroups with the average score of 15.02 (SD $=5.12$ ), the severity of cravings varied insignificantly (17.94, $\mathrm{SD}=8.35, \mathrm{~F}=2.363, \mathrm{df}=2, p=0.096$ ), and there were significant differences in patterns of drinking (see Table 1). The average number of SD consumed weekly was $34.37(\mathrm{SD}=35.13)$, the average number of drinking 
Table 1 Baseline characteristics of study participants

\begin{tabular}{|c|c|c|c|c|c|}
\hline Characteristic & $\mathrm{AHC}^{\mathrm{a}}(n=169)$ & $\mathrm{CH}^{\mathrm{b}}(n=49)$ & $\mathrm{FHT}^{\mathrm{c}}(n=27)$ & Test statistic & All subjects $(n=246)$ \\
\hline \multicolumn{6}{|l|}{$\overline{S e x}, \%$} \\
\hline Men & $63.3 \%$ & $51.0 \%$ & $44.4 \%$ & $x^{2}=4.94, d f=2, p=0.08$ & $58.8 \%$ \\
\hline Women & $36.7 \%$ & $49.0 \%$ & $55.6 \%$ & & $41.2 \%$ \\
\hline Age, mean (SD) & $45.42(11.37)$ & $46.80(13.83)$ & $44.63(11.75)$ & $\mathrm{F}=0.364, \mathrm{df}=2, p=0.695$ & $45.61(11.75)$ \\
\hline Smokers, \% & $51.8 \%$ & $61.2 \%$ & $40.7 \%$ & $x^{2}=3.02, d f=2, p=0.22$ & $52.4 \%$ \\
\hline Cannabis users ${ }^{d}, \%$ & $70.6 \%$ & $77.6 \%$ & $59.3 \%$ & $x^{2}=2.81, d f=2, p=0.25$ & $70.7 \%$ \\
\hline AUD history, years, mean (SD) & $14.29(11.65)$ & $15.56(12.60)$ & $16.52(12.88)$ & $\mathrm{F}=0.47, \mathrm{df}=2, p=0.625$ & $14.85(11.99)$ \\
\hline \multicolumn{6}{|l|}{ Drinking patterns: } \\
\hline $\mathrm{SD} / \mathrm{W}$, mean (SD) & $37.28(37.27)$ & $20.96(26.34)$ & $41.78(26.86)$ & $\mathrm{F}=4.271, \mathrm{df}=2, p=0.015$ & $34.37(35.13)$ \\
\hline SD/d, mean (SD) & $6.59(5.38)$ & $4.31(4.46)$ & $7.89(3.43)$ & $\mathrm{F}=4.509, \mathrm{df}=2, p=0.012$ & $6.24(5.16)$ \\
\hline HDD/w, mean (SD) & $3.68(3.13)$ & $2.60(2.95)$ & $4.06(2.57)$ & $\mathrm{F}=2.481, \mathrm{df}=2, p=0.086$ & $3.50(3.07)$ \\
\hline $\mathrm{DD} / \mathrm{w}$, mean $(\mathrm{SD})$ & $4.11(2.99)$ & $3.14(2.92)$ & $5.11(2.25)$ & $\mathrm{F}=3.325, \mathrm{df}=2, p=0.038$ & $4.00(2.96)$ \\
\hline PACS, mean (SD) & $17.73(8.95)$ & $16.90(7.10)$ & $21.07(5.86)$ & $\mathrm{F}=2.363, \mathrm{df}=2, p=0.096$ & $17.94(8.35)$ \\
\hline MDD history, years, mean (SD) & $13.47(11.37)$ & $9.71(8.51)$ & $11.53(9.66)$ & $\mathrm{F}=1.22, \mathrm{df}=2, p=0.299$ & $12.39(10.61)$ \\
\hline QIDS, mean (SD) & $15.03(5.23)$ & $14.39(5.07)$ & $16.41(4.22)$ & $\mathrm{F}=1.185, \mathrm{df}=2, p=0.307$ & $15.02(5.12)$ \\
\hline
\end{tabular}

${ }^{\mathrm{a}} \mathrm{AHC}$ Academic health centre

${ }^{\mathrm{b}} \mathrm{CH}$ Community hospital

${ }^{C_{F}}$ FHT Family Health Team

${ }^{d}$ Cannabis users were defined as those who used cannabis at least once in the past year

days per week was $4.00(\mathrm{SD}=2.96)$, and the average number of standard drinks per drinking day was 6.24 $(\mathrm{SD}=5.16)$. Overall, the lowest alcohol consumption was reported in patients receiving treatment in community hospitals and the highest in family health teams. The only drinking-related parameter that did not differ significantly between settings was the number of heavy drinking days per week $(3.50, \mathrm{SD}=3.07$, on average in the whole sample).

\section{Treatment parameters}

Average treatment duration varied between 99.31103.11 days in community settings and 134.09 in AHC, which was significantly longer $(\mathrm{F}=3.527, \mathrm{df}=2, p=$ 0.031) than treatment at FHT. There were significant differences between settings in terms of number of physician visits with an average of 7.26 visits across the whole cohort $(\mathrm{F}=4.183, \mathrm{df}=2, p=0.016)$. The largest number was observed in community hospitals, followed by FHT and the lowest in academic settings. There were no significant differences in the number of psychotherapy sessions $(\mathrm{F}=1.209, \mathrm{df}=2, p=0.300)$.

\section{Clinical outcomes}

The data on clinical outcomes are presented in Table 2 . One hundred seventy-four (70.7\%) of patients completed their treatment, with some insignificant variation between sites $\left(x^{2}=3.35, \mathrm{df}=2, p=0.19\right)$. On average, there were statistically significant and clinically meaningful reductions in the number of drinking days per week (1.81, $t=8.78, p<0.001)$, number of heavy drinking days per week $(2.32, t=10.95, \mathrm{p}<0.001)$, average number of SD per drinking day $(3.23, t=8.65, \mathrm{p}<0.001)$ and weekly alcohol consumption $(22.19, t=9.53, \mathrm{p}<0.001)$. The drinking patterns changed the most in academic settings with the only parameter significantly varying between settings being $\mathrm{DD} / \mathrm{w}(2.12, \mathrm{~F}=3.511, \mathrm{df}=2, p=0.032)$. The cohort overall demonstrated significant and meaningful reduction in severity of cravings (Reduction in PACS score of 4.42, $t=8.63, \mathrm{p}<0.001)$ and depressive symptoms (reduction of QIDS score of $4.25, t=11.26$, $p<0.001)$.

\section{Determinants of clinical improvement}

Only the reductions in number of $\mathrm{SD} / \mathrm{w}$ and $\mathrm{DD} / \mathrm{w}$ were significantly associated with the format of therapy - individual therapy was associated with better outcomes $(\beta=$ 9.785, 95\%CI: $0.371-19.199, p=0.042$ and $\beta=1.051$, 95\%CI: $0.220-1.882, p=0.013)$. Correlations between the type of setting and clinical outcomes were in line with the descriptive analyses - treatment in a community hospital and being a patient of FHT was associated with less pronounced reduction in drinking $(\beta=-11.957,95 \% \mathrm{CI}$ : $-0.446--23.468, \mathrm{p}=0.042$ and $\beta=-1.678,95 \% \mathrm{CI}$ : -0.199 $-1.908, p=0.026$, respectively).

\section{Discussion}

The study demonstrated that the ICP paradigm is capable of ensuring high treatment retention rates in complex clinical populations and in a variety of settings. 
Table 2 Summary of the clinical outcomes in the ICP patients

\begin{tabular}{|c|c|c|c|c|c|c|}
\hline Outcome & $\mathrm{AHC}^{\mathrm{a}}(n=169)$ & $\mathrm{HC}^{\mathrm{b}}(n=49)$ & $\mathrm{FHT}^{c}(n=27)$ & Test statistic & $\begin{array}{l}\text { All subjects } \\
(n=246)\end{array}$ & $\begin{array}{l}\text { Base vs. EoT } \\
\text { (paired t-test) }^{d}\end{array}$ \\
\hline Completion rate, $\%$ & $74.1 \%$ & $65.3 \%$ & $59.3 \%$ & $x^{2}=3.35, d f=2, p=0.19$ & $70.7 \%$ & - \\
\hline Treatment duration, mean (SD) & $134.09(106.51)$ & $99.31(43.26)$ & $103.11(30.23)$ & $\mathrm{F}=3.527, \mathrm{df}=2, p=0.031$ & $123.76(92.36)$ & \\
\hline Number of physician visits, mean (SD) & $7.65(4.19)$ & $5.88(2.64)$ & $6.89(3.30)$ & $\mathrm{F}=4.183, \mathrm{df}=2, p=0.016$ & $7.26(3.89)$ & \\
\hline Number of therapy sessions, mean (SD) & $10.95(6.03)$ & $12.45(4.47)$ & $11.41(6.54)$ & $\mathrm{F}=1.209 \mathrm{df}=2, p=0.300$ & $11.30(5.82)$ & \\
\hline \multicolumn{7}{|l|}{ Changes in clinical parameters } \\
\hline Reduction in SD/w, mean (SD) & $25.35(37.06)$ & $13.39(26.01)$ & $16.08(24.03)$ & $\mathrm{F}=2.407, \mathrm{df}=2, p=0.093$ & $22.19(34.46)$ & $\mathrm{t}=9.53, p<0.001$ \\
\hline Reduction in SD/d, mean (SD) & $3.57(6.09)$ & $2.08(4.05)$ & $3.03(2.93)$ & $\mathrm{F}=1.251, \mathrm{df}=2, p=0.288$ & $3.23(5.56)$ & $\mathrm{t}=8.65, p<0.001$ \\
\hline Reduction in HDD/w, mean (SD) & $2.63(3.34)$ & $2.06(2.98)$ & $1.11(2.64)$ & $\mathrm{F}=2.107, \mathrm{df}=2, p=0.124$ & $2.39(3.24)$ & $\mathrm{t}=10.95, p<0.001$ \\
\hline Reduction in DD/w, mean (SD) & $2.12(3.16)$ & $1.23(2.85)$ & $0.44(1.86)$ & $\mathrm{F}=3.511, \mathrm{df}=2, p=0.032$ & $1.81(3.05)$ & $\mathrm{t}=8.78, p<0.001$ \\
\hline Reduction in PACS, mean (SD) & $4.57(8.29)$ & $4.27(7.38)$ & $3.78(6.35)$ & $\mathrm{F}=0.128, \mathrm{df}=2, p=0.880$ & $4.42(7.88)$ & $\mathrm{t}=8.63, p<0.001$ \\
\hline Reduction in QIDS, mean (SD) & $4.17(6.22)$ & $4.74(4.94)$ & $3.77(3.84)$ & $\mathrm{F}=0.262, \mathrm{df}=2, p=0.77$ & $4.25(5.76)$ & $\mathrm{t}=11.26, p<0.001$ \\
\hline
\end{tabular}

${ }^{a} A H C$ Academic health centre

${ }^{\mathrm{b}} \mathrm{HC}$ Community hospital

${ }^{\mathrm{C}}$ FHT Family Health Team

${ }^{d}$ the difference between the baseline and the end-of-treatment parameters for the whole sample

Also, it yielded significant and meaningful clinical improvement in patterns of drinking, severity of cravings and severity of depressive symptoms. Despite the differences in baseline characteristics of patients receiving treatment in the three major types of settings the observed reductions in patterns of drinking, severity of cravings and depressive symptoms were quite similar with slightly better improvements observed in academic settings, likely due to higher availability of clinical resources and higher level of training and specialization of personnel. Also, individual format of therapy and academic settings were associated with significantly better improvement in specific aspects of drinking patterns.

These findings corroborate our preliminary reports on ICP - at the pilot stage, with a small sample or patients $(n=28)$ we reported $80 \%$ retention rate at 12 weeks, which is in line with our current definition of ICP completion, and a tendency of reduction in depressive symptoms and alcohol consumption [8]. Later on, in a larger sample of $n=81$ patients restricted to CAMH only we have demonstrated that ICP patients have significantly higher retention rates in comparison to a convenience sample of matched historical controls ( $81.5 \%$ vs.30.9\%) as well as significant and clinically meaningful reduction in depressive symptoms, cravings and alcohol consumption [9]. Thus, with the expansion of the pathway to a variety of settings and despite rather rapid implementation model the ICP still yielded good clinical outcomes comparable to those presented in literature [25].

As the ICP was created and tailored to specific settings and clinical population there are no data on pathways of the same design. At the same time our data are in line with the findings from several randomized clinical trials (RCT) testing effectiveness of several antidepressants and anti-craving medications in depressed alcoholics $[11-13,26]$. Out of these the most relevant would be the study of Pettinati and colleagues testing the effectiveness of a combination of naltrexone and sertraline in similar population [12]. Despite less rigorous design and no financial incentives our study yielded retention rates higher than in that RCT (70.7\% vs. $57.1 \%)$. At the same time the effect sizes for all clinical outcomes in our cohort were significantly smaller than the ones observed in that trial.

In addition to clinical trials of pharmaceuticals there are at least two clinical trials investigating the effects of structured CBT-based clinical pathways [27, 28]. The studies were smaller ( $n=73$ and $n=37$, respectively), utilized only psychotherapy, but at the same time yielded clinical data comparable to our findings.

In addition, the comparisons to existing literature are complicated due to several methodological limitations of our study, specifically, absence of control group, non-randomized observational design as well as heterogeneity of clinical settings. While the latter aspect of our study allowed to illustrate the impact of the clinical setting on treatment outcomes, the limitations warrant further research aimed to compare the ICP-based treatment to TAU or other treatment models. In addition, the data collection is based on chart review, which though being thorough still leads to a substantial amount of missed data.

Nevertheless, the overall outcomes of the study were positive and corroborate both our previous findings and the results of several studies with similar treatment protocols. Also, as the study is based on a project whose main goal was to broadly implement the ICP protocol in a variety of clinical settings with minimalistic eligibility 
criteria, we can assume that similar results may be expected in future adaptation and implementation of the ICP protocol in other provinces of Canada as well as internationally.

\section{Conclusion}

The study demonstrated that ICP is a feasible and effective treatment for concurrent AUD and MDD that delivers meaningful clinical improvement in a variety of settings. There was a significant variation in baseline patient characteristics and to some degree - in specific clinical outcomes depending on the setting and format of therapy. Controlled study is needed to properly compare the treatment outcomes between ICP model and treatment as usual as well as to further explore the role of various factors on treatment outcomes.

\section{Additional file}

Additional file 1: The DA VINCI Pharmacotherapy Algorithm. The Supplement contains a description of the DA VINCI Pharmacotherapy Algorithm the way it was presented to all clinical sites. Specifically, the algorithm outlines the medications used for treatment of both AUD and MDD as well as the timelines, rules and clinical scales used to justify the medication dose change or switch to another medication. (PDF $112 \mathrm{~kb}$ ).

\section{Abbreviations}

AHC: Academic Health Centre; ANOVA: Analysis of Variance; ARTIC: Adopting Research to Improve Care; AUD: Alcohol Use Disorder; CAMH: Centre for Addiction and Mental Health; CBT: Cognitive Behavior Therapy;

$\mathrm{CH}$ : Community Hospital; DA VINCl: Depression and Alcoholism - Validating an Integrated Care Initiative; DD/w: Number of drinking days per week; FHT: Family Health Team; PACS: Penn Alcohol Craving Scale; QIDS: Quick Inventory of Depressive Symptomatology; QIDS-SR16: Quick Inventory of Depressive Symptomatology - Self-Rated, 16-item; RCT: Randomized Clinical Trial; REB: Research ethics board; SD: Standard deviation; SD/DD: Number of standard drinks per drinking day; SD/w: Number of standard drinks consumed on weekly basis; HDD/w: Number of heavy drinking days per week; ICP: Integrated Care Pathway; MDD: Major Depressive Disorder TAU Treatment as usual

\section{Acknowledgements}

The authors would like to acknowledge the work of Steve Perrotta, Amanda Carpenter, Leena Rahman, and Muna Osman who performed the chart review. Also, we would like to acknowledge the work of a number of clinicians, scientists and administrators involved in creation and implementation of the ICP and directly involved it patient care including the ICP MDAD working group, CAMH ICP program personnel and CAMH AMS clinicians and administrators. Also, we would like to thank our colleagues from all clinical sites for their contribution to implementation of the pathway and their dedication to provision of excellent clinical care to the ICP patients.

\section{Funding}

Two authors, Dr. Samokhvalov and Ms.Awan, are co-leads of the DA VINCI project which was supported by the Adopting Research to Improve Care (ARTIC) Program, a proven model that accelerates the spread and implementation of evidence-based health care interventions across Ontario. ARTIC is co-led by the Council of Academic Hospitals of Ontario (CAHO) and Health Quality Ontario (HQO), and is funded by the Ministry of Health and Long-term Care. The grant was exclusively covering implementation aspects and no research activities were funded, including this study and this paper. Neither $\mathrm{CAHO}$, nor $\mathrm{HQO}$ had any influence on the manuscript preparation or the way the data are being presented and interpreted by the authors.

\section{Availability of data and materials}

The datasets used and/or analysed during the current study are available from the corresponding author on reasonable request.

\section{Authors' contributions}

AVS was the Co-Lead for the DA VINCI project and the Principal Investigator for this study, he designed the study, organized data collection and analyzes, drafted and finalized the manuscript. CP and JR have contributed substantially to study conceptualization, data analyses and manuscript preparation. SA was the Co-lead of the DA VINCI Project and substantially contributed to data collection, interpretation and manuscript preparation. BLF, PV and TG have substantially contributed to the interpretation of clinical data and manuscript preparation. All authors have reviewed and approved the final manuscript for submission.

\section{Ethics approval and consent to participate}

The DA VINCI project was a clinical implementation project. As such, patients receiving treatment through the DA VINCI project were not required to provide consent for participation in a research project. The data for this study were obtained through a clinical chart review approved by the Centre for Addiction and Mental Health Research Ethics Board (Protocol \#053-2016).

\section{Competing interests}

Dr. George has served on Data Safety and Monitoring Boards for Novartis, and has had a grant and contract funding from Pfizer in the past 2 years. Dr. Le Foll has received grant and salary support from Pfizer and Bioprojet. He received speaker fees or has been consultant for Richter Pharmaceuticals, Lundbeck, Ethypharm, Mettrum and Allergan. Drs. George, Le Foll and Rehm held multiple grants from $\mathrm{ClHR}$, NIH and other organizations. None of these activities are related to this study, thus the authors report no conflicts of interest. The authors alone are responsible for the content and writing of this paper.

\section{Publisher's Note}

Springer Nature remains neutral with regard to jurisdictional claims in published maps and institutional affiliations.

\section{Author details}

${ }^{1}$ Addictions Division, Centre for Addiction and Mental Health (CAMH), 33 Russell Street, Office T519, Toronto, ON M5S 2S1, Canada. ${ }^{2}$ Institute for Medical Science, University of Toronto, Toronto, ON, Canada. ${ }^{3}$ Institute for Mental Health Policy Research, CAMH, Toronto, ON, Canada. ${ }^{4}$ Division of Brain and Therapeutics, Department of Psychiatry, University of Toronto, Toronto, ON, Canada. ${ }^{5}$ Campbell Family Mental Health Research Institute, Toronto, ON, Canada. ' Division of Adult Psychiatry and Health Systems, Department of Psychiatry, University of Toronto, Toronto, ON, Canada. ${ }^{7}$ Dalla Lana School of Public Health, University of Toronto, Toronto, ON, Canada. ${ }^{8}$ Institute for Clinical Psychology and Psychotherapy, Technische Universität Dresden, Dresden, Sachsen, Germany. ${ }^{9} \mathrm{WHO}$ Collaborating Centre on Mental Health and Addiction, Toronto, ON, Canada.

Received: 2 January 2018 Accepted: 29 May 2018

Published online: 13 June 2018

\section{References}

1. Awan S, Samokhvalov AV, Aleem N, Hendershot CS, Irving JA, Kalvik A, Lefebvre L, Le Foll B, Quilty L, Voore P: Development and implementation of an ambulatory integrated care pathway for major depressive disorder and alcohol dependence. Psychiatr Serv (Washington, DC) 2015: https://doi. org/10.1176/appi.ps.

2. Pearson C, Janz T, Ali J. Mental and substance use disorders in Canada. In: Health at a Glance. Ottawa: Statistics Canada; 2013.

3. Patten SB, Wang JL, Williams JV, Currie S, Beck CA, Maxwell CJ, El-Guebaly N. Descriptive epidemiology of major depression in Canada. Can J Psychiatr. 2006;51(2):84-90.

4. Currie SR, Patten SB, Williams JV, Wang J, Beck CA, El-Guebaly N, Maxwell C. Comorbidity of major depression with substance use disorders. Can J Psychiatr. 2005;50(10):660-6.

5. Whiteford HA, Degenhardt L, Rehm J, Baxter AJ, Ferrari AJ, Erskine HE, Charlson FJ, Norman RE, Flaxman AD, Johns N, Burstein R, Murray CJ, Vos T. Global burden of disease attributable to mental and substance use 
disorders: findings from the global burden of disease study 2010. Lancet. 2013;382(9904):1575-86.

6. Samokhvalov AV, Popova S, Room R, Ramonas M, Rehm J. Disability associated with alcohol abuse and dependence. Alcohol Clin Exp Res. 2010;34(11):1871-8.

7. Beaulieu S, Saury S, Sareen J, Tremblay J, Schutz CG, McIntyre RS, Schaffer A. The Canadian network for mood and anxiety treatments (CANMAT) task force recommendations for the management of patients with mood disorders and comorbid substance use disorders. Ann Clin Psychiatry : Official J Am Acad Clin Psychiatrists. 2012;24(1):38-55.

8. Samokhvalov AV, Awan S, Voore P. Early outcomes of the integrated care pathway for concurrent major depressive disorder and alcohol dependence. Eur Psychiatry. 2015;30(Supplement 1):1088.

9. Samokhvalov A, Awan S, George T, Irving J, Le Foll B, Perrotta S, Probst C, Voore P, Rehm J. Integrated care pathway for co-occurring major depressive and alcohol use disorders: outcomes of the first two years. Am J Addict. 2017;

10. Diagnostic and Statistical Manual of Mental Disorders. DSM-5. 5th ed. Arlington, VA: American Psychiatric Association; 2013.

11. Kranzler HR, Mueller T, Cornelius J, Pettinati HM, Moak D, Martin PR, Anthenelli R, Brower KJ, O'Malley S, Mason BJ, et al. Sertraline treatment of co-occurring alcohol dependence and major depression. J Clin Psychopharmacol. 2006;26(1):13-20

12. Pettinati HM, Oslin DW, Kampman KM, Dundon WD, Xie H, Gallis TL, Dackis CA, O'Brien CP. A double-blind, placebo-controlled trial combining sertraline and naltrexone for treating co-occurring depression and alcohol dependence. Am J Psychiatry. 2010;167(6):668-75.

13. Cornelius JR, Salloum IM, Ehler JG, Jarrett PJ, Cornelius MD, Perel JM, Thase ME, Black A. Fluoxetine in depressed alcoholics. A double-blind, placebocontrolled trial. Arch Gen Psychiatry. 1997;54(8):700-5.

14. Anton RF, O'Malley SS, Ciraulo DA, Cisler RA, Couper D, Donovan DM, Gastfriend DR, Hosking JD, Johnson BA, LoCastro JS, et al. Combined pharmacotherapies and behavioral interventions for alcohol dependence: the COMBINE study: a randomized controlled trial. Jama. 2006;295(17):2003-17.

15. Trivedi MH, Rush AJ, Crismon ML, Kashner TM, Toprac MG, Carmody TJ, Key T, Biggs MM, Shores-Wilson K, Witte B, et al. Clinical results for patients with major depressive disorder in the Texas medication algorithm project. Arch Gen Psychiatry. 2004;61(7):669-80

16. Guo T, Xiang YT, Xiao L, Hu CQ, Chiu HF, Ungvari GS, Correll CU, Lai KY, Feng L, Geng Y, et al. Measurement-based care versus standard Care for Major Depression: a randomized controlled trial with blind raters. Am J Psychiatry. 2015;172(10):1004-13.

17. Rush AJ, Trivedi MH, Ibrahim HM, Carmody TJ, Arnow B, Klein DN, Markowitz JC, Ninan PT, Kornstein S, Manber R, et al. The 16-item quick inventory of depressive symptomatology (QIDS), clinician rating (QIDS-C), and self-report (QIDS-SR): a psychometric evaluation in patients with chronic major depression. Biol Psychiatry. 2003;54(5):573-83.

18. Kavanagh DJ, Statham DJ, Feeney GF, Young RM, May J, Andrade J, Connor JP. Measurement of alcohol craving. Addict Behav. 2013;38(2):1572-84.

19. Butt P, Beirness D, Cesa F, Gliksman L, Paradis C, Stockwell T. Alcohol and health in Canada: a summary of evidenceand guidelines for low-risk drinking. Ottawa, ON: Canadian Centre on Substance Abuse; 2012.

20. Riper H, Andersson G, Hunter SB, de Wit J, Berking M, Cuijpers P. Treatment of comorbid alcohol use disorders and depression with cognitivebehavioural therapy and motivational interviewing: a meta-analysis. Addiction. 2014;109(3):394-406

21. R Development Core Team. R: A language and environment for statistical computing. Vienna: R Foundation for Statistical Computing; 2008.

22. Gupta SK. Intention-to-treat concept: a review. Pers Clin Res. 2011;2(3):109-12.

23. Little RJ, D'Agostino R, Cohen ML, Dickersin K, Emerson SS, Farrar JT, Frangakis C, Hogan JW, Molenberghs G, Murphy SA, et al. The prevention and treatment of missing data in clinical trials. N Engl J Med. 2012;367(14):1355-60.

24. Samokhvalov AV, Awan S, George TP, Inving J, Le Foll B, Perrotta S, Probst C, Voore $\mathrm{P}$, Rehm J. Integrated care pathway for co-occurring major depressive and alcohol use disorders: outcomes of the first two years. Am J Addict. 2017;26(6):602-9.

25. Rotter T, Kinsman L, James E, Machotta A, Gothe H, Willis J, Snow P, Kugler J. Clinical pathways: effects on professional practice, patient outcomes, length of stay and hospital costs. Cochrane Database Syst Rev. 2010;3:Cd006632.

26. Adamson SJ, Sellman JD, Foulds JA, Frampton CM, Deering D, Dunn A, Berks J, Nixon L, Cape G. A randomized trial of combined citalopram and naltrexone for nonabstinent outpatients with co-occurring alcohol dependence and major depression. J Clin Psychopharmacol. 2015;35(2):143-9.
27. Hunter SB, Watkins KE, Hepner KA, Paddock SM, Munjas B, Osilla KC, Perry S. Treating depression and substance use: a randomized controlled trial. J Subst Abus Treat. 2012;43(2):137-51.

28. Morley KC, Baillie A, Leung S, Sannibale C, Teesson M, Haber PS, Specialized Integrated I. Treatment for comorbid anxiety, depression and alcohol dependence better than treatment as usual in a public hospital setting? Alcohol Alcohol (Oxford, Oxfordshire). 2016;51(4):402-9.

\section{Ready to submit your research? Choose BMC and benefit from:}

- fast, convenient online submission

- thorough peer review by experienced researchers in your field

- rapid publication on acceptance

- support for research data, including large and complex data types

- gold Open Access which fosters wider collaboration and increased citations

- maximum visibility for your research: over $100 \mathrm{M}$ website views per year

At BMC, research is always in progress.

Learn more biomedcentral.com/submissions 